\title{
The Chromatic Index of a Graph Whose Core has Maximum Degree 2
}

\author{
S. Akbari* \\ Department of Mathematical Sciences \\ Sharif University of Technology \\ Tehran, Iran \\ School of Mathematics \\ Institute for Research in Fundamental Sciences (IPM) \\ P.O. Box 19395-5746 \\ Tehran, Iran \\ s_akbari@sharif.edu \\ M. Ghanbari \\ Department of Mathematics \\ K. N. Toosi University of Technology \\ P.O. Box 16315-1618 \\ Tehran, Iran \\ School of Mathematics \\ Institute for Research in Fundamental Sciences (IPM) \\ P.O. Box 19395-5746 \\ Tehran, Iran \\ marghanbari@gmail.com \\ M. $\mathrm{Kano}^{\dagger}$ \\ Department of Computer and Information Sciences \\ Ibaraki University \\ Hitachi, Ibaraki, Japan \\ kano@mx.ibaraki.ac.jp \\ M.J. Nikmehr \\ Department of Mathematics \\ K. N. Toosi University of Technology \\ P.O. Box 16315-1618 \\ Tehran, Iran \\ nikmehr@kntu.ac.ir
}

Submitted: Feb 18, 2011; Accepted: Mar 6, 2012; Published: Mar 19, 2012

Mathematics Subject Classifications: 05C15, 05C70 


\begin{abstract}
Let $G$ be a graph. The core of $G$, denoted by $G_{\Delta}$, is the subgraph of $G$ induced by the vertices of degree $\Delta(G)$, where $\Delta(G)$ denotes the maximum degree of $G$. A $k$-edge coloring of $G$ is a function $f: E(G) \rightarrow L$ such that $|L|=k$ and $f\left(e_{1}\right) \neq f\left(e_{2}\right)$ for all two adjacent edges $e_{1}$ and $e_{2}$ of $G$. The chromatic index of $G$, denoted by $\chi^{\prime}(G)$, is the minimum number $k$ for which $G$ has a $k$-edge coloring. A graph $G$ is said to be Class 1 if $\chi^{\prime}(G)=\Delta(G)$ and Class 2 if $\chi^{\prime}(G)=\Delta(G)+1$. In this paper it is shown that every connected graph $G$ of even order and with $\Delta\left(G_{\Delta}\right) \leq 2$ is Class 1 if $\left|G_{\Delta}\right| \leq 9$ or $G_{\Delta}$ is a cycle of order 10 .
\end{abstract}

Keywords: chromatic index, edge coloring, class 1, core of a graph

\title{
1 Introduction
}

All graphs considered in this paper are finite, undirected, with no loops or multiple edges. Let $G$ be a graph. Then $V(G)$ and $E(G)$ denote the vertex set and the edge set of $G$, respectively. The number of vertices of $G$ is called the order of $G$ and denoted by $|G|$. Also, $\Delta(G)$ and $\delta(G)$ denote the maximum degree and the minimum degree of $G$, respectively. The core of $G$, denoted by $G_{\Delta}$, is the subgraph of $G$ induced by all vertices of degree $\Delta(G)$. We denote the cycle of order $n$ by $C_{n}$. Let $H$ be a subgraph of $G$. For a vertex $u$ of $H, d_{H}(u)$ denotes the degree of $u$ in $H$, and for every vertex $v$ of $G, N_{H}(v)$ denotes $N_{G}(v) \cap V(H)$, where $N_{G}(v)$ is the neighborhood of $v$ in $G$.

A matching in a graph $G$ is a set of pairwise non-adjacent edges, and a 1-factor is a matching which covers $V(G)$. A component $H$ of $G$ is called an odd component if $H$ has odd order, and the number of odd components of $G$ is denoted by $\operatorname{odd}(G)$. For a subset $X \subseteq V(G)(Y \subseteq E(G)), G-X(G-Y)$ denotes the graph obtained from $G$ by deleting all vertices (edges) of $X(Y)$, respectively. Moreover, for a subgraph $H$ of $G$, by $G-H$ we mean the induced subgraph on $V(G)-V(H)$.

A $k$-edge coloring of a graph $G$ is a function $f: E(G) \longrightarrow L$ such that $|L|=k$ and $f\left(e_{1}\right) \neq f\left(e_{2}\right)$ for all two adjacent edges $e_{1}$ and $e_{2}$ of $G$. A graph $G$ is $k$-edge colorable if $G$ has a $k$-edge coloring. The chromatic index of $G$, denoted by $\chi^{\prime}(G)$, is the minimum number $k$ for which $G$ has a $k$-edge coloring. For a general introduction to the edge coloring, the interested reader is referred to [10].

A celebrated result due to Vizing [21] states that for every graph $G, \Delta(G) \leq \chi^{\prime}(G) \leq$ $\Delta(G)+1$. A graph $G$ is said to be Class 1 if $\chi^{\prime}(G)=\Delta(G)$ and Class 2 if $\chi^{\prime}(G)=\Delta(G)+1$. Moreover, a connected graph $G$ is called critical if it is Class 2 and $G-e$ is Class 1 for every edge $e \in E(G)$. A graph $G$ is called overfull if $|E(G)|>\left\lfloor\frac{|V(G)|}{2}\right\rfloor \Delta(G)$. It is easy to see that, if $G$ is overfull, then $G$ is Class 2. For more information about overfull graphs see [12]. In [19] it was proved that there is no critical connected graph $G$ of even order with $\left|G_{\Delta}\right| \leq 5$.

${ }^{*}$ The research was in part supported by a grant from IPM (No. 90050212).

${ }^{\dagger}$ Supported by Grant-in-Aid for Scientific Research of Japan. 
Let $H, Q$ and $R$ be subgraphs of $G$. We denote the number of edges of $H$ with one end point in $Q$ and another end point in $R$ by $e_{H}(Q, R)$. For a subset $S \subseteq V(G)$, we denote the induced subgraph of $G$ on $S$ by $\langle S\rangle_{G}$.

Classifying a graph into Class 1 and Class 2 is a difficult problem in general (indeed, NP hard), even when restricted to the class of graphs with maximum degree 3 (see [17]). As a consequence, this problem is usually considered on classes of graphs with particular classes of cores. One possibility is to consider a graph whose core has a simple structure (see $[3,4,7,9,11,13,14,15,16,22]$ ). Vizing [22] proved that, if $G_{\Delta}$ has no edge, then $G$ is Class 1. Fournier [11] generalized Vizing's result by proving that, if $G_{\Delta}$ contains no cycle, then $G$ is Class 1 . Thus a necessary condition for a graph to be Class 2 is to have a core containing cycles. Hilton and Zhao $[14,15]$ considered the problem of classifying graphs whose cores are a disjoint union of cycles. Only a few such graphs are known to be Class 2. These include the overfull graphs and the graph $P^{*}$, which is obtained from the Petersen graph by removing one vertex and has order 9 . Furthermore, they posed the following conjecture.

Conjecture 1. Let $G$ be a connected graph such that $\Delta\left(G_{\Delta}\right) \leq 2$. Then $G$ is Class 2 if and only if $G$ is overfull, unless $G \neq P^{*}$.

In [6], the following theorem was proved:

Theorem 2. Let $G$ be a connected graph with $\left|G_{\Delta}\right|=3$. Then $G$ is Class 2 if and only if for some integer $n, G$ is obtained from $K_{2 n+1}$ by removing $n-1$ independent edges.

An edge cut is a set of edges whose removal produces a subgraph with more components than the original graph. So a $k$-edge-connected graph has no edge cut of size $k-1$.

Two following results provide some conditions under which a graph $G$ with $\left|G_{\Delta}\right|=4$ is Class 1.

Theorem 3. [5] Let $G$ be a 2-edge-connected graph of even order with $\left|G_{\Delta}\right|=4$. Then $G$ is Class 1 .

Theorem 4. [5] Let $3 \leq r \leq 4$ be an integer and $G$ be an $(r-2)$-edge-connected graph of order $2 n+1$ with $\left|G_{\Delta}\right| \leq r$. Then $G$ is Class 2 if and only if $|E(G)| \geq n \Delta(G)+1$.

Theorem 5. [20] Let $G$ be a critical connected graph with $\Delta(G) \geq 3$. Further suppose that $G$ has $2 n+1 \geq 7$ vertices and $\left|G_{\Delta}\right|=5$. Then $|E(G)|=n \Delta(G)+1$.

The following useful result, which follows from Vizing's Adjacency Lemma [8], is given in Schrijver's homepage [18, p.1765].

Theorem 6. Suppose $k$ is a natural number. Let $v$ be a vertex of a graph $G$ such that $v$ and all its neighbors have degree at most $k$, while at most one neighbor has degree precisely $k$. Then $G$ is $k$-edge colorable if $G-\{v\}$ is $k$-edge colorable.

The previous theorem implies the following well-known result which is due to Fournier.

Theorem 7. [11] If $G_{\Delta}$ is a forest, then $G$ is Class 1. 
Theorem 8. [15] Let $G$ be a connected graph of Class 2 and $\Delta\left(G_{\Delta}\right) \leq 2$. Then the following statements hold.

(i) $G$ is critical;

(ii) $\delta\left(G_{\Delta}\right)=2$;

(iii) $\delta(G)=\Delta(G)-1$, unless $G$ is an odd cycle.

Theorem 9. [15] Let $G$ be a critical connected graph. Then every vertex of $G$ is adjacent to at least two vertices of $G_{\Delta}$.

Theorem 10. [1] Let $G$ be a connected graph with $\Delta\left(G_{\Delta}\right) \leq 2$. Suppose that $G$ has an edge cut of size at most $\Delta(G)-2$ which is a matching or a star. Then $G$ is Class 1 .

A connected graph is called unicyclic if it contains precisely one cycle.

Theorem 11. [1] Let $G$ be a connected graph. If every component of $G_{\Delta}$ is a unicyclic graph or a tree and $G_{\Delta}$ is not a disjoint union of cycles, then $G$ is Class 1.

Theorem 12. [1] Let $G$ be a connected graph of even order. If $\Delta\left(G_{\Delta}\right) \leq 2$ and $\left|G_{\Delta}\right|$ is odd, then $G$ is Class 1 .

Now, we are in a position to prove our main theorem.

Theorem 13. Let $G$ be a connected graph of even order and with $\Delta\left(G_{\Delta}\right) \leq 2$. If $\left|G_{\Delta}\right| \leq 9$ or $G_{\Delta}=C_{10}$, then $G$ is Class 1 .

Proof. For simplicity, let $\Delta=\Delta(G)$. The proof is by induction on $\Delta+|G|$. First note that if $\delta\left(G_{\Delta}\right) \leq 1$ or $\delta(G)<\Delta-1$ or there exists a vertex $x \in V(G)$ such that $\left|N_{G_{\Delta}}(x)\right| \leq 1$, then by Theorems 8 and $9, G$ is Class 1 and we are done. Thus, one can easily assume that $G_{\Delta}$ is a disjoint union of cycles, $\delta(G)=\Delta-1$ and

$$
\left|N_{G_{\Delta}}(x)\right| \geq 2 \quad \text { for every } x \in V(G) .
$$

By (1), we find that $2\left(|G|-\left|G_{\Delta}\right|\right) \leq e_{G}\left(G_{\Delta}, G-G_{\Delta}\right)=(\Delta-2)\left|G_{\Delta}\right|$, and so

$$
|G| \leq \frac{\Delta\left|G_{\Delta}\right|}{2} \leq 5 \Delta
$$

Moreover, if $\left|G_{\Delta}\right|$ is odd, then by Theorem 12, $G$ is Class 1. Thus we can assume that

$\left|G_{\Delta}\right|$ is even, $G_{\Delta}$ is a disjoint union of cycles and $\left|G_{\Delta}\right| \leq 8$ or $G_{\Delta}=C_{10}$.

Note that since $G_{\Delta}$ is a disjoint union of cycles, $\Delta \geq 2$. If $\Delta=2$, then by the connectivity of $G, G$ is a cycle of even order and so $G$ is Class 1 . If $\Delta=3$, then since $|G|$ is even, by Theorem 2 , the assertion is proved. So we may assume that $\Delta \geq 4$. If $G$ has an edge cut of size at most 2 , then by Theorem $10, G$ is Class 1 and we are done. Thus we can suppose that $G$ is 3-edge connected. First we prove the following claim.

Claim 14. G has a 1-factor. 
To the contrary, by Tutte's 1-factor Theorem [2, p.44] and by the assumption that $G$ is of even order, there exists a non-empty subset $T \subseteq V(G)$ such that $\operatorname{odd}(G-T)>|T|$. Let $m=\operatorname{odd}(G-T)$. Since $|G|$ is even, we have $m \equiv|T|(\bmod 2)$, which implies that $m \geq|T|+2$. First assume $T=\{u\}$. Then there exists a component $D$ of $G-T$ such that $e_{G}(u, D) \leq \Delta-2$ by $m \geq 3$. So by Theorem 10, $G$ is Class 1 and we are done. Thus we may assume $|T| \geq 2$.

Let $B_{1}, \ldots, B_{c}$ (big) and $S_{1}, \ldots, S_{d}$ (small) be the odd components of $G-T$ such that $\left|B_{i}\right| \geq \Delta$ for every $1 \leq i \leq c$ and $\left|S_{j}\right| \leq \Delta-1$ for every $1 \leq j \leq d$, where $m=c+d$. Since $|T| \leq m-2$,

$$
|T| \leq c+d-2
$$

Also, since $G$ is 3-edge connected,

$$
e_{G}\left(T, B_{i}\right) \geq 3 \quad \text { for every } \quad 1 \leq i \leq c .
$$

For every $1 \leq j \leq d$, since $1 \leq\left|S_{j}\right| \leq \Delta-1=\delta(G)$, the following hold:

$$
\begin{aligned}
e_{G}\left(T, S_{j}\right) & =\sum_{x \in V\left(S_{j}\right)} e_{G}(T, x) \\
& \geq\left(\delta(G)-\left(\left|S_{j}\right|-1\right)\right)\left|S_{j}\right| \\
& \geq\left(\Delta-\left|S_{j}\right|\right)\left|S_{j}\right| \\
& \geq \Delta-1 .
\end{aligned}
$$

Let $q=\left|T \cap V\left(G_{\Delta}\right)\right|$ and $r=\left|E\left(\langle T\rangle_{G}\right) \cap E\left(G_{\Delta}\right)\right|$. Since $G_{\Delta}$ is a 2-regular graph of order at most 10, the number of edges of $G_{\Delta}$ joining $T$ to $V(G)-T$ satisfies

$$
2 q-2 r=e_{G_{\Delta}}(T, G-T) \leq 2\left(\left|G_{\Delta}\right|-q\right) \leq 2(10-q) .
$$

Hence

$$
q \leq 5+\frac{r}{2}
$$

Since $\left|N_{G_{\Delta}}(x)\right| \geq 2$ for every $x \in V(G),\left|B_{j}\right| \geq \Delta$ and since $G$ is 3-edge connected, we obtain that

$$
e_{G}\left(T, B_{j}\right) \geq \begin{cases}3 & \text { if }\left|V\left(B_{j}\right) \cap V\left(G_{\Delta}\right)\right| \geq 2 \\ \Delta+1 & \text { if }\left|V\left(B_{j}\right) \cap V\left(G_{\Delta}\right)\right|=1 \\ 2 \Delta & \text { otherwise }\end{cases}
$$

Let $c_{0}, c_{1}$ and $c_{2}$ be the number of components $B_{j}$ 's such that $\left|V\left(B_{j}\right) \cap V\left(G_{\Delta}\right)\right|=0$, $\left|V\left(B_{j}\right) \cap V\left(G_{\Delta}\right)\right|=1$ and $\left|V\left(B_{j}\right) \cap V\left(G_{\Delta}\right)\right| \geq 2$, respectively. It is easy to see that $c_{2} \leq 3$ by $\left|G_{\Delta}\right| \leq 10$. Moreover, $c=c_{0}+c_{1}+c_{2}$ and

$$
\begin{aligned}
e_{G}\left(T, B_{1} \cup \cdots \cup B_{c}\right) & \geq 3 c_{2}+(\Delta+1) c_{1}+2 \Delta c_{0} \\
& =(\Delta-1) c-(\Delta-4) c_{2}+2 c_{1}+(\Delta+1) c_{0} .
\end{aligned}
$$


Obviously, using (6) and (9), we have

$$
\begin{aligned}
& q-2 r+|T|(\Delta-1) \\
= & q \Delta-2 r+(|T|-q)(\Delta-1) \\
\geq & e_{G}\left(T, B_{1} \cup \cdots \cup B_{c} \cup S_{1} \cup \cdots \cup S_{d}\right) \\
\geq & (\Delta-1) c-(\Delta-4) c_{2}+2 c_{1}+(\Delta+1) c_{0}+(\Delta-1) d .
\end{aligned}
$$

This implies that

$$
(|T|-c-d)(\Delta-1)+q-2 r+(\Delta-4) c_{2}-2 c_{1}-(\Delta+1) c_{0} \geq 0 .
$$

On the other hand, by (4) and (7), we obtain that

$$
\begin{aligned}
& (|T|-c-d)(\Delta-1)+q-2 r+(\Delta-4) c_{2}-2 c_{1}-(\Delta+1) c_{0} \\
\leq & -2(\Delta-1)+5-\frac{3 r}{2}+(\Delta-4) c_{2}-2 c_{1}-(\Delta+1) c_{0} .
\end{aligned}
$$

Hence, if $c_{2} \leq 2$, then

$$
(|T|-c-d)(\Delta-1)+q-2 r+(\Delta-4) c_{2}-c_{1}-(\Delta+1) c_{0}<0 .
$$

This contradicts (12). Thus, one can assume that $c_{2}=3$ by $c_{2} \leq 3$. If $c_{0} \geq 1$, then similarly (14) holds by (13), and we get a contradiction. So, $c_{0}=0$. We shall show that

$$
c=c_{2}=3 \text { and } V\left(G_{\Delta}\right) \subseteq T \cup\left(\cup_{i=1}^{3} B_{i}\right) .
$$

Suppose, to the contrary, that there exists a component $D$ of $G-\left(T \cup\left(\cup_{i=1}^{3} B_{i}\right)\right)$ such that $\left|V(D) \cap V\left(G_{\Delta}\right)\right| \geq 1$. Now, since $c_{2}=3$ and $\left|G_{\Delta}\right| \leq 10$, we have $q \leq 3$. Note that if $q \leq 1$, then $G_{\Delta}$ is a disjoint union of at least four cycles, a contradiction. If $q=2$, then $G_{\Delta}$ consists of at least three cycles and $\left|G_{\Delta}\right| \geq 11$, a contradiction. If $q=3$, then $G_{\Delta}$ consists of at least two cycles and $\left|G_{\Delta}\right| \geq 11$, a contradiction. Therefore (15) holds.

By (15), $G_{\Delta}$ passes through exactly three components of $G-T$. By (11) and (15),

$$
q-2 r+|T|(\Delta-1) \geq 9+(\Delta-1) d .
$$

Now, if $d \geq|T|$, then by $\Delta \geq 4$,

$$
q-2 r \geq 9+(d-|T|)(\Delta-1) \geq 9,
$$

which contradicts (7). Thus, we can suppose that $d \leq|T|-1$. Now, by $c=3$ and (4),

$$
d=|T|-1 \text {. }
$$

By (5), (8), (10) and (15), we obtain that

$$
q-2 r+|T|(\Delta-1) \geq 9+\sum_{j=1}^{d}\left(\Delta-\left|S_{j}\right|\right)\left|S_{j}\right| .
$$


Thus

$$
(|T|-d)(\Delta-1)+q-2 r-9-\sum_{j=1}^{d}\left(\left(\Delta-\left|S_{j}\right|\right)\left|S_{j}\right|-(\Delta-1)\right) \geq 0 .
$$

On the other hand, by (7) and (17), we find that

$$
\begin{aligned}
& (|T|-d)(\Delta-1)+q-2 r-9-\sum_{j=1}^{d}\left(\left(\Delta-\left|S_{j}\right|\right)\left|S_{j}\right|-(\Delta-1)\right) \\
\leq & \Delta-10+5-\frac{3}{2} r-\sum_{j=1}^{d}\left(\left(\Delta-\left|S_{j}\right|\right)\left|S_{j}\right|-(\Delta-1)\right) .
\end{aligned}
$$

If $\Delta=4$, then $\left|S_{j}\right|=1$ or 3 and so $\left(\Delta-\left|S_{j}\right|\right)\left|S_{j}\right|-(\Delta-1)=0$ for all $j$. Thus

$$
\begin{aligned}
& \Delta-10+5-\frac{3}{2} r-\sum_{j=1}^{d}\left(\left(\Delta-\left|S_{j}\right|\right)\left|S_{j}\right|-(\Delta-1)\right) \\
= & 4-10+5-\frac{3}{2} r \\
= & -1-\frac{3}{2} r<0 .
\end{aligned}
$$

This contradicts (18). Hence $\Delta \geq 5$. If $3 \leq\left|S_{k}\right| \leq \Delta-2$ for some $k$, then $-((\Delta-$ $\left.\left.\left|S_{k}\right|\right)\left|S_{k}\right|-(\Delta-1)\right) \leq-\Delta+3$. So,

$$
\begin{aligned}
& \Delta-10+5-\frac{3}{2} r-\sum_{j=1}^{d}\left(\left(\Delta-\left|S_{j}\right|\right)\left|S_{j}\right|-(\Delta-1)\right) \\
\leq & \Delta-10+5-\frac{3}{2} r-\Delta+3 \\
= & -2-\frac{3}{2} r<0 .
\end{aligned}
$$

This contradicts (18). Therefore, since $\left|S_{j}\right|$ is odd, we conclude that

$$
\Delta \geq 5, \quad \text { and } \quad\left|S_{j}\right|=1 \text { or } \Delta-1 \text { for every } 1 \leq j \leq d .
$$

By (6), (15), (17) and by the fact that every vertex $u$ of $T$ is adjacent to at least two vertices of $G_{\Delta}$, we find that

$$
|T|(\Delta-2) \geq e_{G}\left(T, \cup_{j=1}^{d} S_{j}\right) \geq d(\Delta-1)=(|T|-1)(\Delta-1) .
$$

This concludes that $|T| \leq \Delta-1$. 
First assume that $\left|S_{k}\right|=1$ for some $k, 1 \leq k \leq d$. Let $V\left(S_{k}\right)=\{w\}$. Then since $d_{G}(w)=\Delta-1,|T| \geq \Delta-1$. Thus $|T|=\Delta-1$ and $d=\Delta-2$ by (17). It follows from (2) that

$$
4 \Delta-1+\sum_{j=1}^{\Delta-2}\left|S_{j}\right| \leq|T|+\left|B_{1}\right|+\left|B_{2}\right|+\left|B_{3}\right|+\sum_{j=1}^{d}\left|S_{j}\right| \leq|G| \leq 5 \Delta .
$$

Hence $\left|S_{j}\right|=1$ for all $1 \leq j \leq d$ by (19). Let $S_{j}=\left\{x_{j}\right\}, 1 \leq j \leq d$. Then $N_{G}\left(x_{j}\right)=T$ for every $j$, and so for every vertex $u \in T, e_{G}\left(u, \cup_{j=1}^{d} S_{j}\right)=d=\Delta-2$, which implies $d_{G}(u)=\Delta$ as $\left|N_{G_{\Delta}}(u)\right| \geq 2$. So $T \subset V\left(G_{\Delta}\right)$ and $e_{G}\left(u, \cup_{i=1}^{3} B_{i}\right) \leq 2$ for every $u \in T$. Now, since $c_{2}=3, q \leq 4$ and $e_{G}\left(T, B_{i}\right) \geq 3$, we obtain

$$
3 \times 3 \leq e_{G}\left(T, B_{1} \cup B_{2} \cup B_{3}\right) \leq|T| \times 2=q \times 2 \leq 8 .
$$

This is a contradiction.

Next, suppose that $\left|S_{j}\right|=\Delta-1$ for every $1 \leq j \leq d$. Then it follows from (1) and (15) that $e_{G}\left(T, S_{j}\right) \geq 2\left|S_{j}\right|=2 \Delta-2$ for every $1 \leq j \leq d$ and $e_{G}\left(u, \cup_{j=1}^{d} S_{j}\right) \leq \Delta-2$ for every $u \in T$. Then similar to (20), we have

$$
|T|(\Delta-2) \geq e_{G}\left(T, \cup_{j=1}^{d} S_{j}\right) \geq(|T|-1)(2 \Delta-2),
$$

and so $|T|=1$. This is a contradiction with $|T| \geq 2$. Consequently the proof of the claim is complete.

Now, let $M$ be a 1-factor of $G$, and $H=G-M$. Then $\Delta(H)=\Delta-1, \delta(H)=\delta(G)-1$, $V\left(H_{\Delta}\right)=V\left(G_{\Delta}\right), H_{\Delta} \subseteq G_{\Delta}, \delta\left(H_{\Delta}\right) \geq \delta\left(G_{\Delta}\right)-1=1$, and by $(1)$,

$$
\left|N_{H}(v) \cap V\left(H_{\Delta}\right)\right| \geq 1 \quad \text { for every } v \in V(H) .
$$

It is obvious that if $H$ is Class 1 , then so is $G$. Thus we can assume that $H$ is Class 2 . In particular, $H$ is not connected since otherwise by induction hypothesis, $H$ is Class 1.

Claim 15. $G_{\Delta}$ consists of exactly two disjoint cycles.

By $(3), G_{\Delta}$ is a disjoint union of cycles. Now, suppose that $G_{\Delta}$ is a cycle. If $\delta\left(H_{\Delta}\right)=1$, then by Theorem 7, every component of $H$ is Class 1 , and so is $H$, a contradiction. Hence we may assume that $H_{\Delta}$ is a cycle. By (21), $H$ is connected, a contradiction. Thus $G_{\Delta}$ is a disjoint union of at least two cycles. By (3), $G_{\Delta}$ is a disjoint union of two cycles. Therefore the claim is proved.

Now, we want to show that $H$ has a component whose core is a cycle. First note that by (21), every component of $H$ contains at least one vertex of $H_{\Delta}$. If the core of each component of $H$ has a vertex of degree 1, then by Theorem 8, each component of $H$ is Class 1 and so $H$ is Class 1 , a contradiction. Thus $H$ contains at least one component, say $Q$, whose core is a disjoint union of cycles. If $Q_{\Delta}$ contains exactly two cycles, then by (21) $Q=H$. Thus $H$ is connected, a contradiction. Therefore $Q_{\Delta}$ is a cycle. 
Let $R=H-Q$. Clearly, since $|G|$ is even, $|Q| \equiv|R|(\bmod 2)$. First assume that $Q$ has even order. Then by induction hypothesis $Q$ is Class 1 . Moreover, if the core of $R$ is not a cycle, then by Theorem $7, R$ is Class 1 . If the core of $R$ is a cycle, then $R$ is connected, and since $|R|$ is even, by induction hypothesis $R$ is Class 1 , and so is $H$, a contradiction. Therefore we may assume that both $Q$ and $R$ have odd orders. Since $H$ is Class 2 and by the fact that if the core of $R$ is not a cycle, then $R$ is Class 1 , we may assume that $Q$ is Class 2 .

Let $C_{k}=Q_{\Delta}$ be a cycle of order $k \in\{3,4,5\}$. We need the following claims.

Claim 16. $|Q|=\Delta-3+k$.

Let $|Q|=2 h+1$. Since $Q$ is Class 2 and $\Delta(Q)=\Delta-1 \geq 3$, by Theorems 8 and 10 , $Q$ is critical and 2-edge connected. Moreover, if $Q_{\Delta}=C_{5}$, then $|Q| \geq 7$. Since $Q_{\Delta}=C_{k}$, $k \in\{3,4,5\}$, it follows from Theorems 4,5 and 8 that

$$
\frac{k(\Delta-1)+(2 h+1-k)(\Delta-2)}{2}=|E(Q)| \geq h(\Delta-1)+1 .
$$

Thus $|Q|=2 h+1 \leq \Delta-3+k$. On the other hand,

$$
|Q| \geq\left|C_{k}\right|+\left|N_{Q}(x) \cap V\left(Q-C_{k}\right)\right|=k+\Delta-3 \quad \text { for every } x \in V\left(C_{k}\right)
$$

since $Q_{\Delta}=C_{k}$ and $\Delta(Q)=\Delta-1$. Thus $|Q|=\Delta-3+k$ and $N_{Q}(x) \supseteq V(Q)-V\left(C_{k}\right)$ for every $x \in V\left(C_{k}\right)$. Therefore the claim is proved, and the following (22) holds.

$$
x y \in E(Q) \quad \text { for every } \quad x \in V\left(Q_{\Delta}\right) \text { and } y \in V(Q)-V\left(Q_{\Delta}\right) .
$$

Let $F=\left\{u_{1} v_{1}, \ldots, u_{t} v_{t}\right\}$ be the set of those edges of $M$ such that $u_{i} \in V(Q)$ and $v_{i} \in V(R)$ for every $1 \leq i \leq t$. We show that $V\left(Q_{\Delta}\right) \subseteq\left\{u_{1}, \ldots, u_{t}\right\}$. To the contrary, let $x \in V\left(Q_{\Delta}\right) \backslash\left\{u_{1}, \ldots, u_{t}\right\}$. Since $M$ covers all vertices of $G$, there exists a vertex $y \in V(Q)-\left\{u_{1}, \ldots, u_{t}\right\}$ such that $x y \in M$. If $y \in V\left(Q_{\Delta}\right)$, then since $x \in V\left(Q_{\Delta}\right), Q_{\Delta}$ is not a cycle, a contradiction. If $y \notin V\left(Q_{\Delta}\right)$, then $x y \in M$ contradicts (22). Since $Q_{\Delta}=C_{k}$, without loss of generality, we may assume that

$$
\begin{gathered}
V\left(Q_{\Delta}\right)=\left\{u_{1}, \ldots, u_{k}\right\} \subseteq\left\{u_{1}, \ldots, u_{t}\right\} \\
\text { where } u_{i} u_{i+1} \in E\left(Q_{\Delta}\right) \text { for all } 1 \leq i \leq k-1 \text { and } u_{k} u_{1} \in E\left(Q_{\Delta}\right) .
\end{gathered}
$$

Moreover, since $G_{\Delta}$ is an induced subgraph of $G$ and $Q_{\Delta}=C_{k}$, we have

$$
u_{i} v_{i} \notin E\left(G_{\Delta}\right) \quad \text { for } i=1, \ldots, t \text {, }
$$

and

$$
V\left(R_{\Delta}\right) \cap\left\{v_{1}, \ldots, v_{k}\right\}=\emptyset .
$$

Now, we want to give a lower bound for $t=|F|$. First note that if $|F| \leq \Delta-2$, then by Theorem 10, $G$ is Class 1 . Now, suppose that $|F|=\Delta-1$. Let $Q^{\prime}=G-R$ and $R^{\prime}=G-Q$. Add a new vertex $w_{1}$ and join $w_{1}$ to each $u_{i}, 1 \leq i \leq t$, and denote 
the resultant graph by $Q^{\prime \prime}$. Also, do the same thing for $R^{\prime}$ with a new vertex $w_{2}$, and denote the resultant graph by $R^{\prime \prime}$. Since $|G|>\left|R^{\prime \prime}\right|,\left|Q^{\prime \prime}\right|$ and $\Delta(G) \geq \Delta\left(R^{\prime \prime}\right), \Delta\left(Q^{\prime \prime}\right)$, by the induction hypothesis both $Q^{\prime \prime}$ and $R^{\prime \prime}$ have a $\Delta$-edge coloring with colors $\{1, \ldots, \Delta\}$. By a suitable permutation of colors, one may assume that $c\left(w_{1} u_{i}\right)=c\left(w_{2} v_{i}\right)=i$ for $i=1, \ldots, \Delta-1$, where $c(e)$ denotes the color of $e$. Then by assigning color $i$ to each edge $u_{i} v_{i}, i=1, \ldots, \Delta-1$, we obtain a $\Delta$-edge coloring of $G$ and so $G$ is Class 1 .

Hence we can assume that $|F| \geq \Delta$. Now, since $|Q|=\Delta-3+k$ and $k \leq 5$, we have $|Q| \leq \Delta+2$. This implies that

$$
\Delta \leq|F| \leq \Delta+2
$$

By $(22)$ and since $\delta(Q)=\Delta-2$, for every $y \in V(Q)-V\left(Q_{\Delta}\right)$, we have $\Delta-2 \geq$ $d_{Q}(y) \geq k$, which implies

$$
\Delta \geq k+2 .
$$

Now, we want to prove the following claim.

Claim 17. If $\left\{u_{i} u_{j}, v_{i} v_{j}\right\} \subseteq E(G)$ for some $i, j \in\{1, \ldots, t\}$, then $G$ is Class 1 .

Consider $M^{\prime}=\left(M-\left\{u_{i} v_{i}, u_{j} v_{j}\right\}\right) \cup\left\{u_{i} u_{j}, v_{i} v_{j}\right\}$. Let $Q^{\prime}=Q-\left\{u_{i} u_{j}\right\}$ and $R^{\prime}=$ $R-\left\{v_{i} v_{j}\right\}$. We claim that $G^{\prime}=G-M^{\prime}$ is Class 1 . We show that there exists a path which joins a vertex of $Q_{\Delta}^{\prime}$ to a vertex of $R_{\Delta}^{\prime}$ in $G^{\prime}$. First note that since $Q$ is Class 2, by Theorems 8 and 9 , every $v \in V(Q)$ satisfies $\left|N_{Q_{\Delta}}(v)\right| \geq 2$. Thus, $\left|N_{Q_{\Delta}^{\prime}}\left(u_{i}\right)\right| \geq 1$ and $\left|N_{Q_{\Delta}^{\prime}}\left(u_{j}\right)\right| \geq 1$. Moreover, by $(21),\left|N_{R_{\Delta}}(v)\right| \geq 1$ for every $v \in V(R)$. Now, if $v_{j} \notin V\left(R_{\Delta}\right)$, then since $\left|N_{R_{\Delta}}\left(v_{i}\right)\right| \geq 1,\left|N_{R_{\Delta}^{\prime}}\left(v_{i}\right)\right| \geq 1$ which implies that there exists a path which joins a vertex of $Q_{\Delta}^{\prime}$ to a vertex of $R_{\Delta}^{\prime}$ in $G^{\prime}$. If $v_{j} \in V\left(R_{\Delta}\right)$, then there exists a path which joins $v_{j}$ to a vertex of $Q_{\Delta}^{\prime}$ in $G^{\prime}$.

If $R_{\Delta}$ is a cycle, then $G^{\prime}$ is connected and by induction hypothesis, $G^{\prime}$ is Class 1 and so $G$ is Class 1. Otherwise, for every component $K$ of $G^{\prime}, \delta\left(K_{\Delta}\right)=1$ and $\Delta\left(K_{\Delta}\right) \leq 2$. Thus by Theorem 8, $G^{\prime}$ is Class 1 , so is $G$ and the claim is proved.

Now, two cases may be occurred. First suppose that $Q$ and $R$ are Class 2. Then by (3) and since $Q_{\Delta}$ is a cycle, we can suppose that $R_{\Delta}=C_{r}$, for $r=3,4,5$. So, similar to the proof of Claim 16, $|R|=\Delta-3+r$. Now, similar to (23) and with no loss of generality, one can assume that $v_{t} \in V\left(R_{\Delta}\right)$ and so by $(24), u_{t} \notin V\left(Q_{\Delta}\right)$ and $v_{1} \notin V\left(R_{\Delta}\right)$ and so $u_{1} u_{t} \in E(Q)$ and $v_{1} v_{t} \in E(R)$, by (22). By Claim 17, $G$ is Class 1 and we are done.

Next, assume that $Q$ is Class 2 and $R$ is Class 1 . First we prove the following claim.

Claim 18. If $\left|N_{R_{\Delta}}\left(v_{i}\right)\right|+\left|N_{R_{\Delta}}\left(v_{i+1}\right)\right| \leq 3$ for some $1 \leq i \leq k(\bmod k)$, then $G$ is Class 1 .

Without loss of generality, suppose that $\left|N_{R_{\Delta}}\left(v_{1}\right)\right|+\left|N_{R_{\Delta}}\left(v_{2}\right)\right| \leq 3$. First note that if $v_{1} v_{2} \in E(G)$, then by Claim 17, $G$ is Class 1 and we are done. So, suppose that $v_{1} v_{2} \notin$ $E(G)$. By (1) and assumptions, we can assume that $\left|N_{R_{\Delta}}\left(v_{1}\right)\right|=1$ and $\left|N_{R_{\Delta}}\left(v_{2}\right)\right| \leq 2$. Let $N_{R_{\Delta}}\left(v_{1}\right)=\{x\}$. Now, consider $Q-\left\{u_{1} u_{2}\right\}$, add a new vertex $w_{1}$ and join $w_{1}$ to $u_{1}$ and $u_{2}$. Then call the resultant graph by $Q^{\prime}$. Clearly, $\Delta\left(Q^{\prime}\right)=\Delta(Q)=\Delta-1$. Note that 
since $\Delta \geq 4, Q_{\Delta}^{\prime}$ is a path and by Theorem $7, Q^{\prime}$ has a $(\Delta-1)$-edge coloring with colors $\{1, \ldots, \Delta-1\}$. Moreover, we can assume that $c\left(w_{1} u_{1}\right)=1$ and $c\left(w_{1} u_{2}\right)=2$.

Now, add a new vertex $w_{2}$ to $R$, join $w_{2}$ to $v_{1}$ and $v_{2}$ and call the resultant graph by $R^{\prime}$. By (25), $V\left(R_{\Delta}\right) \cap\left\{v_{1}, v_{2}\right\}=\emptyset$ and so $\Delta\left(R^{\prime}\right)=\Delta(R)=\Delta-1$. We claim that $R^{\prime}$ is Class 1. Let $R^{\prime \prime}=R^{\prime}-\left\{v_{1}\right\}$. Thus $d_{R^{\prime \prime}}\left(w_{2}\right)=1$ and $d_{R^{\prime \prime}}(x)=\Delta-2$ which implies that $x \notin V\left(R_{\Delta}^{\prime \prime}\right)$. We claim that every component $K$ of $R^{\prime \prime}$ is Class 1 and so is $R^{\prime \prime}$. If $\delta\left(K_{\Delta}\right) \leq 1$, then by Theorem $11, K$ is Class 1 . If $K_{\Delta}$ is a cycle, then clearly $w_{2} \in V(K)$. Now, by Theorem 8 and since $1=\delta(K)<\Delta(K)-1, K$ is Class 1 . This implies that $R^{\prime \prime}$ is Class 1. Now, by Theorem 6 , since $d_{R}\left(v_{1}\right)=\Delta-1$ and $d_{R}(x)=\Delta-1$ and $R^{\prime \prime}$ is Class $1, R^{\prime}$ has a $(\Delta-1)$-edge coloring with colors $\{1, \ldots, \Delta-1\}$. Moreover, we can assume that $c\left(w_{2} v_{1}\right)=1$ and $c\left(w_{2} v_{2}\right)=2$. Now, color $u_{1} v_{1}$ and $u_{2} v_{2}$ by 1 and 2 , respectively and then color every edge $f \in\left(F-\left\{u_{1} v_{1}, u_{2} v_{2}\right\}\right) \cup\left\{u_{1} u_{2}\right\}$ by $\Delta$ to obtain a $\Delta$-edge coloring of $G$ and the claim is proved.

So, we can assume that

$$
\left|N_{R_{\Delta}}\left(v_{i}\right)\right|+\left|N_{R_{\Delta}}\left(v_{i+1}\right)\right| \geq 4 \text { for } i=1, \ldots, k(\bmod k) .
$$

This implies that

$$
\sum_{i=1}^{k}\left|N_{R_{\Delta}}\left(v_{i}\right)\right| \geq 2 k .
$$

Moreover, since $V\left(G_{\Delta}\right) \cap\left\{u_{k+1}, \ldots, u_{t}\right\}=\emptyset$, (1) yields that $\left|N_{R_{\Delta}}\left(v_{i}\right)\right| \geq 2$ for $i=k+$ $1, \ldots, t$. This implies that

$$
\sum_{i=1}^{t}\left|N_{R_{\Delta}}\left(v_{i}\right)\right| \geq 2 t .
$$

Now, we want to prove the following claim. Let $L=R-\left\{v_{1}, \ldots, v_{t}\right\}$.

Claim 19. Let $u_{i} u_{j} \in E(G)$ for some $i, j \in\{1, \ldots, t\}$ and $x y \in M \cap E(L)$. If $v_{i} x, v_{j} y \in$ $E(G)$, then $G$ is Class 1 .

Consider $M^{\prime}=\left(M-\left\{u_{i} v_{i}, u_{j} v_{j}, x y\right\}\right) \cup\left\{u_{i} u_{j}, v_{i} x, v_{j} y\right\}$. Let $G^{\prime}=G-M^{\prime}$. Now, remove two edges $v_{i} x$ and $v_{j} y$ of $R$ and add $x y$ to the edges of $R$ and call the resultant graph by $R^{\prime}$. By (28) and with no loss of generality, one can assume that $\left|N_{R_{\Delta}}\left(v_{i}\right)\right| \geq 2$. This implies that $v_{i}$ is adjacent to at least one vertex of $R_{\Delta}^{\prime}$. Also, since $Q$ is Class 2 , by Theorems 8 and $9,\left|N_{Q_{\Delta}^{\prime}}\left(u_{i}\right)\right| \geq 1$, where $Q^{\prime}=Q-\left\{u_{i} u_{j}\right\}$. Thus there exists a path which joins one vertex of $Q_{\Delta}^{\prime}$ to a vertex of $R_{\Delta}^{\prime}$. Now, if $G^{\prime}$ is connected, then by induction hypothesis, $G^{\prime}$ is Class 1 and so $G$ is Class 1 . Otherwise, since there exists a path which joins one vertex of $Q_{\Delta}^{\prime}$ to a vertex of $R_{\Delta}^{\prime}$, for every component $K$ of $G^{\prime}, \delta\left(K_{\Delta}\right) \leq 1$ and $\Delta\left(K_{\Delta}\right) \leq 2$. Thus by Theorem $8, K$ is Class 1 and so is $G^{\prime}$. This implies that $G$ is Class 1 and the claim is proved.

By (23), $V\left(Q_{\Delta}\right) \cap\left\{u_{1}, \ldots, u_{t}\right\}=\left\{u_{1}, \ldots, u_{k}\right\}$, where $k=3,4,5$. Now, by (22), $u_{i} u_{j} \in E(Q)$ for $i=1, \ldots, k$ and $j=k+1, \ldots, t$. Note that $d_{Q}\left(u_{i}\right)=\Delta-1$ and $d_{Q}\left(u_{j}\right)=\Delta-2$ for $i=1, \ldots, k$ and $j=k+1, \ldots, t$, respectively. Now, by Claim 16, $u_{i}$ is not adjacent to exactly $k-3$ vertices in the set $\left\{u_{1}, \ldots, u_{t}\right\}$ for $i=1, \ldots, k$. Moreover, 
$u_{j}$ is not adjacent to at most $k-2$ vertices in the set $\left\{u_{1}, \ldots, u_{t}\right\}$ for $j=k+1, \ldots, t$. Note that if $\left\{u_{i} u_{j}, v_{i} v_{j}\right\} \subseteq E(G)$, for some $i, j \in\{1, \ldots, t\}$, then by Claim 17, $G$ is Class 1 and we are done. Thus, we can suppose that for $k=3,4,5$,

$$
\begin{gathered}
\left|N_{R}\left(v_{i}\right) \cap\left\{v_{1}, \ldots, v_{t}\right\}\right| \leq k-3 \text { for } i=1, \ldots, k, \\
\left|N_{R}\left(v_{j}\right) \cap\left\{v_{1}, \ldots, v_{t}\right\}\right| \leq k-2 \text { for } j=k+1, \ldots, t .
\end{gathered}
$$

Since $d_{R}\left(v_{i}\right) \geq \Delta-2$ for $i=1, \ldots, t$, we conclude that for $k=3,4,5$,

$$
\begin{aligned}
& e_{R}\left(v_{i}, L\right) \geq \Delta-k+1 \text { for } i=1, \ldots, k . \\
& e_{R}\left(v_{j}, L\right) \geq \Delta-k \text { for } j=k+1, \ldots, t .
\end{aligned}
$$

Now, two cases may be occurred:

First suppose that $|L| \leq 2 \Delta-2 k+2$. Let $M \cap E(L)=\left\{x_{1} y_{1}, \ldots, x_{m} y_{m}\right\}$. Thus $m \leq \Delta-k+1$. With no loss of generality, suppose that

$$
N_{R}\left(v_{1}\right) \cap V(L)=\left\{x_{1}, \ldots, x_{s+t}, y_{1}, \ldots, y_{s}\right\} .
$$

Thus by (30),

$$
2 s+t \geq \Delta-k+1 \text { for some } s, t \text {. }
$$

Now, if

$$
\left\{x_{1}, \ldots, x_{s}, y_{1}, \ldots, y_{s+t}\right\} \cap\left(N_{R}\left(v_{2}\right) \cap V(L)\right) \neq \emptyset,
$$

then since $u_{1} u_{2} \in E(Q)$ by Claim 19, we are done. So, we can suppose that

$$
N_{R}\left(v_{2}\right) \cap V(L) \subseteq\left\{x_{s+1}, \ldots, x_{m}, y_{s+t+1}, \ldots, y_{m}\right\} .
$$

Thus by (30), (32) and since $|L| \leq 2 \Delta-2 k+2$,

$$
\begin{aligned}
\left|N_{R}\left(v_{2}\right) \cap V(L)\right| & \leq 2 \Delta-2 k+2-(2 s+t) \\
& \leq 2 \Delta-2 k+2-(\Delta-k+1) \\
& =\Delta-k+1 .
\end{aligned}
$$

So, by (30),

$$
N_{R}\left(v_{2}\right) \cap V(L)=\left\{x_{s+1}, \ldots, x_{m}, y_{s+t+1}, \ldots, y_{m}\right\}
$$

and $|L|=2 \Delta-2 k+2$. Now, if

$$
\left\{x_{s+t+1}, \ldots, x_{m}, y_{s+1}, \ldots, y_{m}\right\} \cap\left(N_{R}\left(v_{3}\right) \cap V(L)\right) \neq \emptyset,
$$

then since $u_{2} u_{3} \in E(Q)$ by Claim 19 , we are done. So, by a similar argument as we did for $v_{2}$, we conclude that

$$
N_{R}\left(v_{3}\right) \cap V(L)=\left\{x_{1}, \ldots, x_{s+t}, y_{1}, \ldots, y_{s}\right\}
$$


Now, we do this procedure for $v_{i}, i \leq k$ and so

$$
\begin{cases}N\left(v_{k+1}\right) \subseteq N\left(v_{1}\right) & \text { if } k \text { is even } \\ N\left(v_{k+1}\right) \subseteq N\left(v_{2}\right) & \text { if } k \text { is odd. }\end{cases}
$$

Now, if $s \geq 1$, then with no loss of generality one may assume that there exists an edge $x_{i} y_{i}$ for some $i=1, \ldots, t$ such that

$$
\begin{cases}\left\{v_{1} x_{i}, v_{k+1} y_{i}\right\} \subseteq E(Q) & \text { if } k \text { is even } \\ \left\{v_{2} x_{i}, v_{k+1} y_{i}\right\} \subseteq E(Q) & \text { if } k \text { is odd }\end{cases}
$$

Moreover, by (22), $\left\{u_{1} u_{k+1}, u_{2} u_{k+1}\right\} \subseteq E(Q)$ and so by Claim 19, $G$ is Class 1 . Thus we can suppose that $s=0$ and so

$$
N\left(v_{i}\right) \subseteq\left\{x_{1}, \ldots, x_{m}\right\} \text { for } i=1, \ldots, t .
$$

Now, by pigeonhole principle, (26), (30) and (31), for some $i=1, \ldots, t$,

$$
d_{R}\left(x_{i}\right) \geq \frac{k(\Delta-k+1)+(\Delta-k)^{2}}{\Delta-k+1} .
$$

Now, by $(27), d_{R}\left(x_{i}\right)>\Delta-1$, a contradiction.

Now, suppose that $|L|>2 \Delta-2 k+2$. Note that since $M$ is a 1-factor, $L$ has even order. Thus we can suppose that

$$
|L| \geq 2 \Delta-2 k+4
$$

By (26), let $|F|=\Delta+i$, where $i=0,1,2$. Therefore we find

$$
|R| \geq 3 \Delta-2 k+4+i \text {. }
$$

Now, we want to determine an upper bound for $|R|$. Suppose that $\left|R_{\Delta}\right|=r$. Let $X$ be the set of those vertices of $L-R_{\Delta}$ such that $\left|N_{R_{\Delta}}(x)\right|=1$. So, for every $y \in L-\left(X \cup R_{\Delta}\right)$, $\left|N_{R_{\Delta}}(y)\right| \geq 2$. Note that since $G_{\Delta}$ is a disjoint union of cycles, the minimum degree of the core of every component of $H_{\Delta}$ is at least 1. Thus, for every $w \in V\left(R_{\Delta}\right)$, since $d_{R}(w)=\Delta-1, e_{R}\left(w, R-R_{\Delta}\right) \leq \Delta-2$. Moreover, let $N_{G_{\Delta}}(x)=\left\{v_{x}, w_{x}\right\}$ such that $N_{R_{\Delta}}(x)=\left\{v_{x}\right\}$. Clearly, $|X|=\left|\left\{w_{x} \mid x \in X\right\}\right|$ and so $e_{R}\left(w_{x}, R-R_{\Delta}\right) \leq \Delta-3$. Let $\left|V\left(R_{\Delta}\right) \cap\left\{v_{1}, \ldots, v_{t}\right\}\right|=d$. Now, since $V\left(R_{\Delta}\right) \cap\left\{v_{1}, \ldots, v_{k}\right\}=\emptyset$, by (28), (29) we find that

$$
\begin{aligned}
& 2(t-d)+|X|+2(|R|-(t+|X|+r-d)) \\
\leq & e_{R}\left(R_{\Delta}, R-R_{\Delta}\right) \\
\leq & |X|(\Delta-3)+(r-|X|)(\Delta-2) .
\end{aligned}
$$

This implies that

$$
|R| \leq \frac{r \Delta}{2}
$$


Now, by (34),

$$
3 \Delta-2 k+4+i \leq \frac{r \Delta}{2}
$$

Since $r \in\{3,4,5\}$, this implies that

$$
\Delta \leq \frac{4 k-8-2 i}{6-r}
$$

Now, three cases can be considered:

(i) $r=3$. Since $G_{\Delta}$ has even order, $k \in\{3,5\}$. So, by Claim 16 and since $|Q|$ is odd, $\Delta$ is odd. Now, by (36), $\Delta \leq 4$. Thus $\Delta=4$, a contradiction.

(ii) $r=4$. Since $G_{\Delta}$ has even order, $k=4$. Moreover, by Claim $16,|Q|=\Delta+1$ and so $i=1$. Thus, by (36) we conclude that $\Delta \leq 3$, a contradiction.

(iii) $r=5$. Since $G_{\Delta}$ has even order and $\left|G_{\Delta}\right| \leq 8, k=3$. Moreover, by Claim 16 and since $|Q|$ is odd, $\Delta$ is odd. Now, by (36), $\Delta \leq 3$, a contradiction and the proof is complete.

The following remark states that why the main idea of the proof of Theorem 13 fails for general graphs.

Remark. In [1], it is shown that if $G$ is a connected graph of even order, $\Delta\left(G_{\Delta}\right) \leq 2$ and $\left|G_{\Delta}\right|$ is odd, then $G$ is Class 1 . Thus as we mentioned in the proof of Theorem 13, it suffices to prove the assertion for $\left|G_{\Delta}\right| \leq 8$. We know that if $G$ is Class 2 , then $G_{\Delta}$ is a 2-regular graph and since the number of vertices of $G_{\Delta}$ is small and indeed at most 8 , $G_{\Delta}$ is a disjoint union of at most two cycles. In our proof, first we proved the existence of a 1-factor $M$ in $G$. Next, we considered $G-M$. The worst case was whenever $G-M$ is not connected, one of its components is Class 2 with odd number of vertices, and moreover its core has exactly one cycle. There are useful results in connection to graphs whose cores have order at most 5, see [5],[6],[19] and [20]. Indeed, if $G_{\Delta}$ has order more than 9 in the aforementioned part of the proof of Theorem 13, that component which is Class 2 maybe have a core with more than 5 vertices and there is no good information about the structure of such graphs. Therefore, we have some serious problems to prove Theorem 13 for the graphs with large cores.

Acknowledgments. The authors would like to express their deep gratitude to the referee for her/his fruitful comments.

\section{References}

[1] S. Akbari, D. Cariolaro, M. Chavoshi, M. Ghanbari, S. Zare. Some Criteria for a graph to be Class 1. Discrete Math., to appear.

[2] J.A. Bondy and U. S. R. Murty. Graph Theory with Applications. North Holland, New York, 1976. 
[3] D. Cariolaro, G. Cariolaro. Colouring the petals of a graph. Electronic Journal of Combinatorics, 10:\#R6, 2003.

[4] A.G. Chetwynd and A.J.W. Hilton. A $\Delta$-subgraph condition for a graph to be Class 1. J. Combin. Theory Ser. B, 46:37-45, 1989.

[5] A.G. Chetwynd and A.J.W. Hilton. The chromatic index of graphs with at most four vertices of maximum degree. Congressus Numerantium, 43:221-248, 1984.

[6] A.G. Chetwynd and A.J.W. Hilton. Regular graphs of high degree are 1-factorizable. Proc. London Math. Soc., 50:193-6, 1985.

[7] A.G. Chetwynd and A.J.W. Hilton. 1-factorizing regular graphs of high degree an improved bound. Discrete Math., 75:103-112, 1989.

[8] S.A. Choudum, K.Kayathri. An extension of Vizing's adjacency lemma on edge chromatic critical graphs. Discrete Math., 206:97-103, 1999.

[9] J.K. Dugdale and A.J.W. Hilton. A sufficient condition for a graph to be the core of a Class 1 graph. Combinat. Prob. Comput., 9:97-104, 2000.

[10] S. Fiorini and R.J. Wilson. Edge-colourings of graphs. Research Notes in Mathematics, Pitman, 1977.

[11] J.-C. Fournier. Coloration des aretes dun graphe. Cahiers du CERO (Bruxelles), 15:311-314, 1973.

[12] A.J.W. Hilton. Two conjectures on edge-colouring. Discrete Math., 74:61-64, 1989.

[13] A.J.W. Hilton and C. Zhao. A sufficient condition for a regular graph to be Class 1. J. Graph Theory, 17:701-712, 1993.

[14] A.J.W. Hilton and C. Zhao. On the edge-colouring of graphs whose core has maximum degree two. JCMCC, 21:97-108, 1996.

[15] A.J.W. Hilton and C. Zhao. The chromatic index of a graph whose core has maximum degree two. Discrete Math., 101:135-147, 1992.

[16] D.G. Hoffman. Cores of Class II graphs. J. Graph Theory, 20:397-402, 1995.

[17] I. Holyer. The NP-completeness of edge-colouring. SIAM Journal of Comp., 10:718$720,1981$.

[18] A. Schrijver. Advanced Graph Theory and Combinatorial Optimization, (http://homepages.cwi.nl/ lex/), 2001.

[19] Z. Song and H. P. Yap. Chromatic index critical graphs of even order with five major vertices. Graph and Comb., 21:239-246, 2005.

[20] Z. Song. Chromatic index critical graphs of odd order with five major vertices. J. Combin. Math. Combin. Comput., 41:161-186, 2002.

[21] V.G. Vizing. On an estimate of the chromatic class of a p-graph. (in Russian), Diskret. Analiz., 3:25-30, 1964.

[22] V.G. Vizing. Critical graphs with a given chromatic class (in Russian). Diskret. Analiz, 5:6-17, 1965. 